\title{
Interactive comment on "Concerted measurements of lipids in seawater and on submicron aerosol particles at the Cape Verde Islands: biogenic sources, selective transfer and high enrichments" by Nadja Triesch et al.
}

\section{Anonymous Referee \#1}

Received and published: 15 October 2020

The manuscript presents a very interesting dataset on lipids, investigated in seawater, sea-surface microlayer and submicron aerosol particles at the CVAO - Cape Verde Atmospheric Observatory. Both dissolved and particulate lipids were studied, showing different degrees of enrichment and the partitioning into different classes among the three compartments studied. This is very interesting, and as the authors point out, the lipid composition in seawater, sea-surface microlayer and aerosols highlights that not only autotrophic sources may be responsible for the organic composition of submicron aerosols, but that bacterial activities and further oxidation and photodegradation pro- 
cesses on these atmospheric particles may be responsible for their different organic matter content. This is important to individuate the sources of these materials and possibly, their behavior once in the atmosphere.

The manuscript is well written, just some minor spelling mistakes and wording should be looked at, and it well addresses the scope of ACP. It is also novel as it presents concerted measurements on three compartments, usually studied separately in field work. The scientific approach and methodology is sound and well described, accompanied with an extensive sub-set of supplementary information.

Overall I recommend the publication of the paper, but I suggest some minor issues to be taken into account prior to this.

I think the authors shall include some more information on the importance of this study in the field. I found the manuscript technically correct, but in my opinion it lacks a bit of background information and broader outlook. I have these comments in particular:

What is the importance of PM1 aerosols with respect to other size-distributions? How would you expect lipid classes be different in other size-fractions of marine aerosols?

Why did you chose the glass plate for SML sampling compared to other devices, and can you estimate (if any), biases of the glass-plate method on lipids concentration with respect to other components of this layer?

Line 32 page 5: It is not clear to me why did you analyze $\mathrm{Na}+$ and why you used it to calculate EFaer. Is it related to seawater salts?

In the figures (e.g. figure 1), maybe you can specify in the caption that where there is no_SML you refer to ULW, I suppose.

Lines 7-11, page 11: it is not very clear to me whether in the SML PL were enriched, or $\mathrm{DL}$, as in page 18, line 32, you state that $P L$ are more degraded in the $S M L$, and more stable in the DL and PL in the ULW. This sounds a bit confusing. If PL are enriched in the SML, I would expect them to be more stable then, and less degraded.

Interactive

comment

Printer-friendly version

Discussion paper

C2 
Line 23, page 11: is it possible that the lower enrichment of lipids compared to other OM classes like amino acids is due to biases in the glass plate method?

Line 3 page 12: it is also possible that phytoplankton activity is lower in the SML because of high radiation, thus cells stay preferentially below the SML. I expect that at the Cape Verde Islands solar radiation was quite high. Are there any measurements on this parameter? It would be interesting to see how it can influence the different lipid composition in seawater, SML and PM1. Do you have any data on this? Did you run some tests?

How do you relate the lipid composition of submicron aerosols, that have presumably been in the atmosphere for a longer residence time, to the ambient seawater composition both in ULW and SML, that may rapidly change even during diel cycles? I don't know if it makes sense, but would it be possible to estimate an EFaer based on ULW properties instead of SML components?

Just as a curiosity, would bigger aerosol particles show a more straightforward relation to seawater properties, considering that they may have resided in the atmosphere for a shorter time and travelled over shorter distances?

The PM1 lipid fraction resembled more the DL fraction of seawater, in composition. Would it be possible that by sampling larger aerosols, the lipid composition would have resembled more the PL fraction of seawater instead? If so, could this be because of molecular size?

What are the implications of lipid composition for marine aerosols? Could the different lipid fractions lead to different cloud forming capacities, or aerosol properties concerning optical thickness and radiative effects? Are these properties relevant for the study region, or other marine regions? I think it would be a good addition to the paper to discuss these aspects and broader implications in the conclusion section. 
Interactive comment 